\title{
Cuantificación de biomasa aérea utilizando medidas dasométricas para la Guadua angustifolia Kunth, en la cuenca hidrográfica del río Guarapas municipio de Pitalito Huila.
}

\author{
Gustavo Adolfo Ramírez Córdoba ${ }^{1}$ \\ Ingeniero Agroforestal, Maestría en Sistemas Sostenibles de Producción \\ Universidad Nacional Abierta y a Distancia - UNAD \\ Grupo de Investigación INYUMACIZO, Semillero SIMAC. \\ gustavo.ramirez@unad.edu.co
}

\section{Diana Stefany Molina Calderón ${ }^{2}$}

Estudiante Ingeniería Ambiental Universidad Nacional Abierta y a Distancia - UNAD Grupo de Investigación INYUMACIZO, Semillero SIMAC. distefy95@hotmail.com

\section{William Sneyder Montealegre Rojas ${ }^{3}$}

Estudiante Ingeniería Ambiental

Universidad Nacional Abierta y a Distancia - UNAD

Grupo de Investigación INYUMACIZO, Semillero SIMAC. wil-mon@hotmail.com

\section{RESUMEN}

La Guadua angustifolia Kunth es una especie vegetal abundante en el sur del departamento de Huila, lo que la convierte en un recurso altamente aprovechable y con grandes posibilidades de contribución a problemas sociales, económicos y ambientales de la región. Sin embargo, la investigación realizada con esta especie vegetal en la región apenas está presentando su incremento, gracias al grupo de investigación INYUMACIZO y la UNAD. Para continuar con este proceso, se realizó la cuantificación de la biomasa aérea de la Guadua angustifolia Kunth sobre la cuenca del río Guarapas en Pitalito, como aporte a la cuantificación de captura carbono orgánico en guaduales, dando un resultado final de $82 \mathrm{Ton} / \mathrm{ha}$ de aporte promedio de esta especie.

DOI: https://doi.org/10.22490/ECAPMA.2948 
Palabras Claves: Cambio climático, método destructivo, sostenibilidad.

\begin{abstract}
Guadua angustifolia Kunth is an abundant plant species in the southern department of Huila, which makes it a highly profitable resource with great potential contribution to social, economic and environmental problems in the region. However, research conducted with this plant species in the region is just presenting its increase, thanks to the research group INYUMACIZO and UNAD. To continue this process, quantitation of the biomass of Guadua angustifolia Kunth over basin Guarapas river Pitalito performed as input to the quantification of organic capture carbon guaduales, giving a final result of 82 tons / ha of average contribution of this species.
\end{abstract}

Keywords: Climate change, destructive method, sustainability.

\title{
Introducción
}

La guadua técnicamente es un bambú leñoso o más comúnmente es considerada un pasto gigante, taxonómicamente pertenece a la familia de Poaceae o Gramineae y del cual existen realmente en el mundo cerca de 1400 especies, 435 de ellas nativas de América (Bambú, 2015). De éstas aproximadamente 20 conforman las especies prioritarias de bambú, y dentro de ellas, Colombia tiene una que posee las mejores propiedades físicomecánicas del mundo y extraordinaria durabilidad: La Guadua angustifolia. Esta especie cuenta con culmos erectos que alcanzan alturas hasta de 25 metros y diámetros entre 10 y $25 \mathrm{~cm}$., sus entrenudos tienen paredes hasta de $2 \mathrm{~cm}$. de espesor (Teneche, S.f.), y además se resaltan los diversos beneficios que esta especie vegetal aporta; dentro de ellos la ventaja de reproducirse permanentemente convirtiéndose en un recurso altamente renovable. Así mismo, evita la movilización de tierra y conserva los suelos, además de su gran capacidad para el almacenamiento de agua (Ecohabitar, 2015).

En su defecto la guadua está ligada al concepto de sostenibilidad en la medida que dichos procesos pueden ser más sencillos, económicos y con productos muy competitivos, debido a que la guadua tiene fibras naturales muy fuertes que permiten desarrollar productos industrializados, tales como aglomerados, laminados, pisos, paneles, esteras, pulpa y papel (Bárbaro, 2007; Herrera, 
2008; Camargo et al., 2010), que se podrían ofrecer en el mercado nacional o internacional, compitiendo con el plástico, hierro y concreto, sin mencionar la gran contribución a la capacidad de fijación de CO2, lo cual genera un aporte al control del cambio climático (Mendoza, Cano \& Rojas, 2015). La guadua está asociada a temas importantes como los servicios ecosistémicos (Corredor, Fonseca \& Páez, 2012), la sustentabilidad ambiental (Giraldo \& Valencia, 2010), el turismo (Vinasco, 2017), la valoración de la fauna y la flora (Bonilla \& Caetano, 2013), el trabajo comunitario (Mendoza, Cano \& Rojas, 2015), así como a perspectivas interdisciplinarias para atender la alimentación (Zapata \& Franco, 2014) y formas alternativas de desarrollo rural (Montes \& Sánchez, 2016; Duarte \& Arana-Gutiérrez, 2016)

Es claro afirmar, que otros trabajos realizados a nivel local han aportado a esta investigación como la Formulación Plan prospectivo y estratégico para la consolidación de la cadena productiva de la guadua en la zona sur del Huila Colombia (Cuellar B. Adelaida, Montealegre T. William, Méndez P. Nelly M. 2016), entre otros la guadua cobra una gran importancia ambiental debido a que en el municipio de Pitalito Huila se encuentra una extensión de 347,9 has en guadua (Montealegre, 2014).

El grupo de investigación del macizo colombiano INYUMACIZO (INYUMACIZO, 2012) y el semillero de investigación del macizo colombiano SIMAC han priorizado, dentro de su plan de acción, avanzar en el estudio de la especie natural de flora silvestre Guadua, desde lo local y a lo largo de su proceso de crecimiento ha desarrollado investigaciones tendientes a ese objetivo.

\section{REVISIÓN BIBLIOGRÁFICA}

La Guadua angustifolia Kunth, es una de las especies de Bambú más resistentes, llamado como el acero vegetal, por sus cualidades en el momento de utilizarse bien sea en artesanías menores como en grandes construcciones arquitectónicas, no obstante, brinda unos excelentes servicios ambientales en los cuales reconocemos como un gran retenedor y regulador de agua y fuentes hídricas, protección de suelos, biodiversidad, microclimas, entre otros.

Tomando en cuenta que, en Colombia, los guaduales se presentan principalmente en riberas de los ríos, esto logra que la guadua cumpla un papel excepcional como protector de las cuencas hidrográficas en la que está 
presente, ya que aporta, por medio de su biomasa, a mejorar la textura y estructura del suelo, a acumular agua y liberarla en épocas de escases, y a la protección y sostenibilidad de flora y fauna asociadas a esta especie (Agroempresarial S.A., 2012).

Tabla 1. Clasificación taxonómica de la Guadua angustifolia Kunth

\begin{tabular}{|c|c|}
\hline Nombre Científico & Guadua angustifolia Kunth \\
\hline Reino & Vegetal \\
\hline División & Espermatofitas \\
\hline Subdivisión & Angiospermas \\
\hline Clase & Monocotiledóneas \\
\hline Orden & Glumiflorales \\
\hline Familia & Gramíneas \\
\hline Tribu & Bambusease verae \\
\hline Genero & Guadua \\
\hline Subgénero & Bambusa \\
\hline Especie & Guadua angustifolia \\
\hline \multirow[t]{3}{*}{ Formas } & Guadua Castilla \\
\hline & Guadua Macana \\
\hline & Guadua Cebolla \\
\hline \multirow[t]{2}{*}{ Variedades } & Guadua Bicolor Verde rayada y amarilla \\
\hline & $\begin{array}{ll}\text { Guadua Negra } & \text { El gen determinante no } \\
& \text { se ha adquirido } \\
& \text { totalmente }\end{array}$ \\
\hline
\end{tabular}


Tabla 2. Variables climatológicas óptimas de estación para la guadua

Hábitat

Precipitación

Humedad Relativa

Condiciones de óptimo
De 0 a 2200 m de altitud sobre el nivel del mar Superior a $1200 \mathrm{~mm} / \mathrm{año}$

$75 \%-85 \%$

Fuente: González, y Díaz (2003). Adaptado por Méndez (2014)

Según (Camargo, 2010), la Guadua angustifolia Kunth aporta en promedio, por individuo, una cantidad de $4,5 \mathrm{~kg}$ de biomasa a sus 7 años, de la cual el $74,6 \%$ se encuentra localizada en la biomasa aérea, correspondiente a las hojas, ramas y culmo, y puede aportar desde el 80,1\% (Riaño, Londoño, López, \& Gómez, 2002) hasta el 83\% (Camargo, 2010) del total de la capacidad de fijación de carbono de la guadua.

El cambio climático es la situación más preocupante en los últimos años, debido a que está causando el derretimiento de los polos, el aumento del nivel del mar, cambio de temperaturas en diferentes zonas a nivel mundial, aumento en las actividades sísmicas, entre otras consecuencias (Cambio climático.org, S.f.). El aumento de la temperatura del planeta o cambio climático global y la influencia de los bosques naturales (González et al., 2008) y artificiales (IPCC, 2007) como reguladores de este han sido temas ampliamente discutidos y documentados.

La producción y desarrollo de la guadua presenta una posible alternativa para contribuir al control del cambio climático, ya que diversos estudios, como "Acumulación y predicción de biomasa y carbono en plantaciones de bambú en Costa Rica" (González \& Vargas, 2015) o "Posibilidades de la guadua para la mitigación del cambio climático" (Arango, 2011), demuestran que la guadua presenta características de captación de Dióxido de Carbono excepcionales, y su almacenamiento puede durar hasta por 80 años, además de que presenta una gran resistencia al fuego, debido a su ácido sílico, y retiene el agua y los nutrientes para utilizarlos en épocas de escases, disminuyendo la erosión y reteniendo nutrientes hasta quince veces más que las coberturas compuestas por pasturas (Chará et al., 2010).

El bambú guadua es una alternativa real como sustituto de la madera, de él se pueden obtener industrialmente diferentes productos como laminados, 
aglomerados, columnas, vigas, viguetas, cuartones, tablas, paneles y variedad de productos como artesanías, compostaje, forraje, medicina, carbón, pulpa y energía (Bárbaro, 2007; Herrera, 2008; Camargo et al., 2010).

También se resalta que la Guadua angustifolia Kunth presenta diversas cualidades que le permitirían entrar dentro de los mecanismos de desarrollo limpio y los mercados de carbono, por su alta y constante fijación de carbono en su biomasa (Ríos, S.f.).

\section{METODOLOGÍA}

El trabajo de campo se realizó basado en el método destructivo, que consiste en el aprovechamiento de los individuos para tomar muestras húmedas (Mónaco, Rosa, Santa, Autrán, \& Heguiabehere, 2015), y se desarrolló por medio de las siguientes fases:

Muestra: Se seleccionaron 120 rodales de guadua ubicados en diferentes zonas, que abarcan toda la cuenca del río Guarapas. En cada rodal de guadua se seleccionaron tres guaduas con los que se realizó el trabajo de campo, los rodales de guadua fueron distribuidos por seis rutas (Palestina, Mocoa, Timaná, Saladoblanco, Chillurco, urbano).

Marcación de guaduas: Las guaduas utilizadas como muestra fueron seleccionadas al azar, sin considerar su edad o estado de madurez, tomando en cuenta que su ubicación sea en diferentes puntos del rodal, realizando georreferenciación de los mismos.

Corte: Las guaduas seleccionadas para el muestreo fueron cortadas en el primer o segundo entrenudo, y posteriormente se realizaron medidas dasométricas y se dividieron en tres compartimientos con el fin de realizar mediciones de canutos y densidad de madera. Luego se tomó $1 \mathrm{~kg}$ de muestras de biomasa húmeda, de la siguiente manera: $400 \mathrm{gr}$ de madera, $300 \mathrm{gr}$ de hojas y $300 \mathrm{gr}$ de ramas.

Secado: Las muestras recolectadas fueron introducidas a un horno, previamente pesadas, donde se les realizó un secado a $50^{\circ} \mathrm{C}$ durante 48 horas continuas. Por medio de esto, se determinó el contenido de humedad, lo que permitió determinar la cantidad de biomasa de la Guadua angustifolia Kunth por medio de una serie de ecuaciones, relacionando los datos obtenidos de contenido de humedad, longitudes y circunferencias. 


\section{RESULTADOS}

Inicialmente, el contenido de humedad arrojó un valor promedio de 34\% por individuo, y desagrupando los datos por componente evaluado (culmo, ramas, hojas) se obtienen los siguientes resultados:

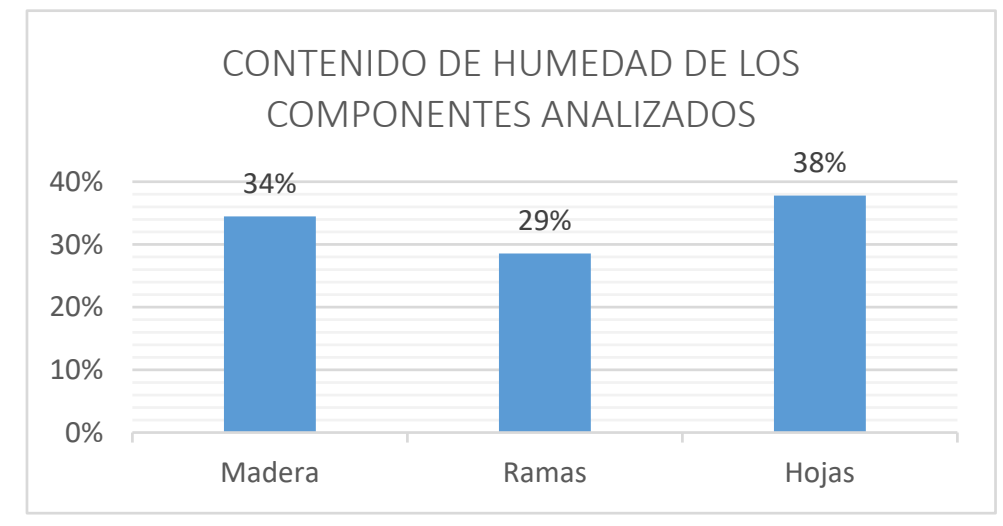

Figura 1. Contenido de humedad por componentes. Fuente: Autores (2018)

En la figura 1, se muestra la comparación entre los tres componentes respecto a su contenido de humedad, demostrando una notable diferencia entre las ramas y las hojas, de un $9 \%$ de diferencia.

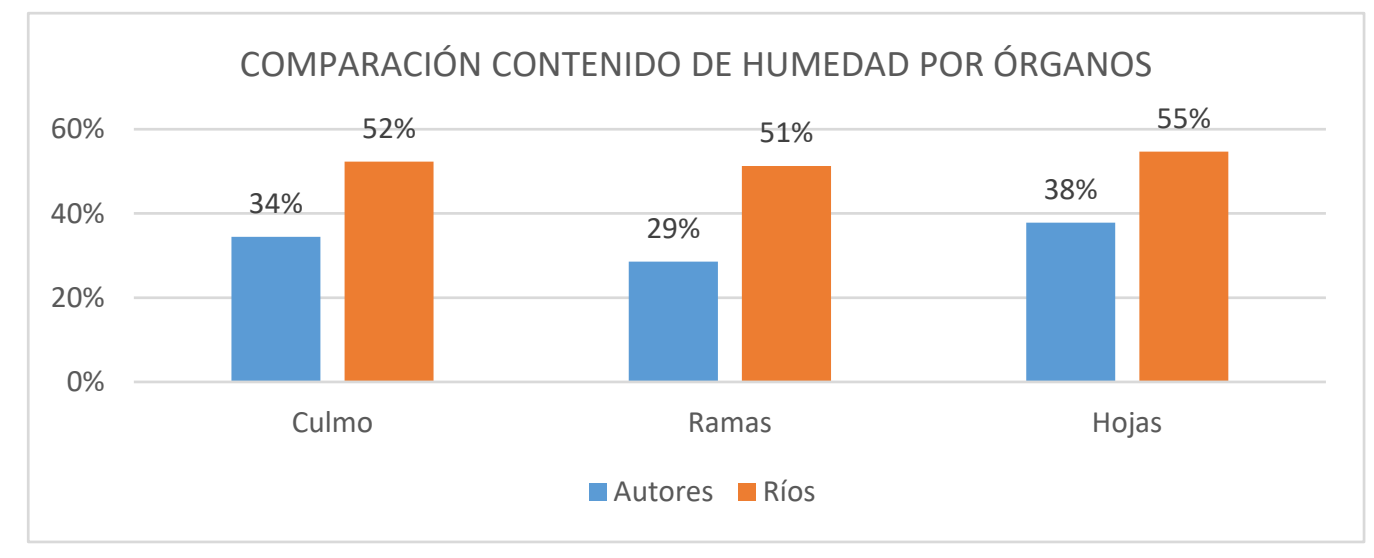

Figura 2. Comparación contenido de humedad. Fuente: Autores (2018)

También se realizó una comparación, con los resultados de un estudio similar realizado por Hormilson Ríos (S.f.) con plantaciones de guadua en México, como se muestra en la figura 2, la cual demuestra una gran diferencia entre 
el contenido de humedad de cada componente evaluado dando resultados muy bajos para la guadua estudiada en este proyecto.

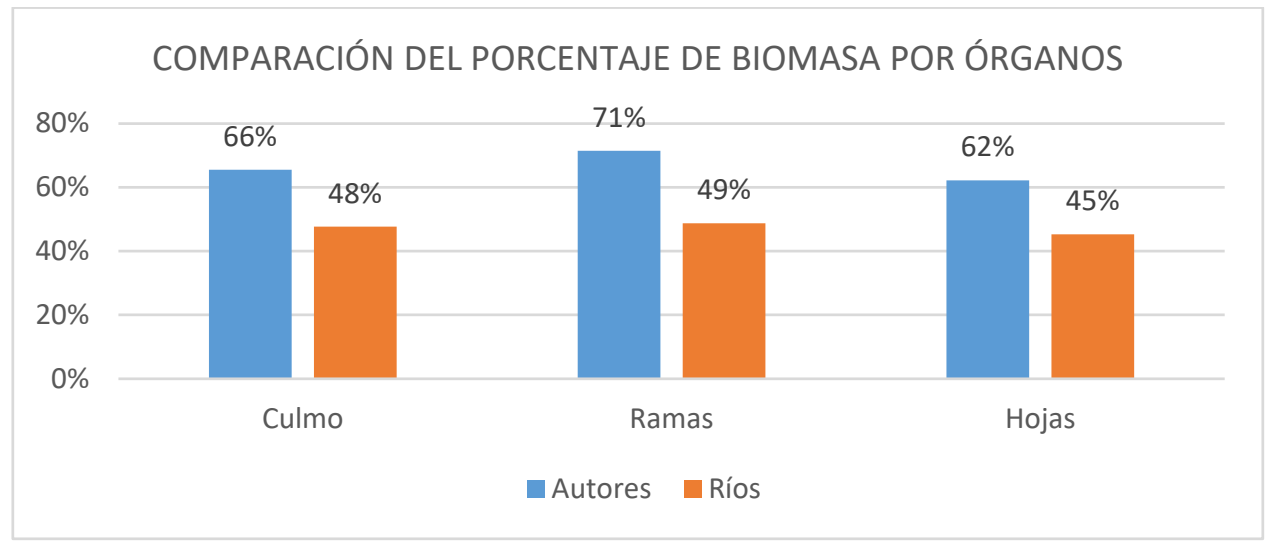

Figura 3. Comparación del porcentaje de biomasa por órganos.

Fuente: Autores (2018)

Otro análisis realizado fue el porcentaje de biomasa por órgano, dando como resultado los valores representados por las columnas azules en la figura 3, además de su comparación con los datos obtenidos en el trabajo de Ríos (S.f.), dando, en esta ocasión, resultados muy favorables respecto al porcentaje de biomasa por guadua.

Tabla 3. Resultados de biomasa seca

\begin{tabular}{|c|c|c|c|c|}
\hline & Culmo & Ramas & Hojas & Total \\
\hline $\begin{array}{l}\text { Promedio biomasa seca } / \mathrm{cm} 3 \\
\text { guadua }\end{array}$ & 15647,32 & 4729,94 & 1582,75 & 21960,01 \\
\hline $\begin{array}{l}\text { Promedio biomasa seca } / \mathrm{m} 3 \\
\text { guadua }\end{array}$ & 0,015 & 0,0047 & 0,0015 & 0,02 \\
\hline Densidad guadua $\mathrm{Kg} / \mathrm{m} 3$ & 687,08 & 687,08 & 687,08 & 687,08 \\
\hline $\begin{array}{l}\text { Promedio biomasa seca } / \mathbf{K g} \\
\text { guadua }\end{array}$ & 10,75 & 3,25 & 1,087 & 15,08 \\
\hline Promedio biomasa seca/Ha cm3 & $\begin{array}{l}85528274, \\
5\end{array}$ & $\begin{array}{l}25853852, \\
8\end{array}$ & $\begin{array}{l}8651315,1 \\
5\end{array}$ & $\begin{array}{l}120033442 \\
, 5\end{array}$ \\
\hline Promedio biomasa seca/Ha m3 & 85,53 & 25,85 & 8,65 & 120,03 \\
\hline Promedio biomasa seca/Ha Kg & 58764,77 & 17763,66 & 5944,14 & 82472,57 \\
\hline Promedio biomasa seca/ha Ton & 58,76 & 17,76 & 5,94 & 82,47 \\
\hline Biomasa seca total CH Ton & 20444,26 & 6179,98 & 2067,97 & 28692,20 \\
\hline
\end{tabular}

Fuente: Autores (2018)

En la tabla 3 se demuestran los resultados de los valores correspondientes a la biomasa seca, es decir, el objetivo del presente trabajo, resaltando los valores de biomasa seca/ha Ton para cada órgano evaluado, y en total, dando como resultado un aporte de 82,47 Ton/ha promedio de biomasa aérea seca, 
y un valor total de 28692,2 Ton para la cuenca hidrográfica del ríos Guarapas, en el municipio de Pitalito.

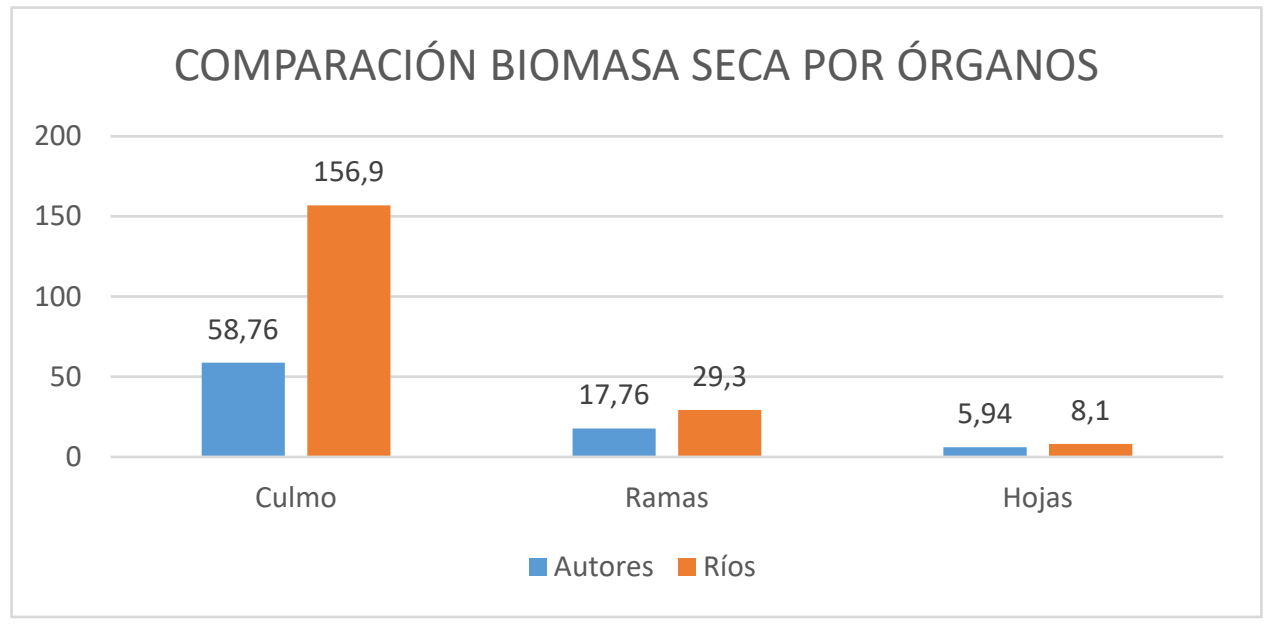

Figura 4. Comparación de biomasa seca por órganos

Fuente: Autores (2018)

A estos valores también se les realizó una comparación con el trabajo de Ríos (S.f.), dando resultados desfavorables, ya que los resultados de Ríos dan unos valores relativamente altos, y tomando en cuenta que la densidad de culmos por hectárea no es muy diferenciada ( 5755 para Ríos, 5466 para el presente estudio), por lo tanto, una posible explicación es que Ríos tomó en consideración la edad y el estado de madurez, ya que era una plantación, y para nuestro caso, se utilizaron guaduales naturales.

\section{Biomasa Ton/Ha}

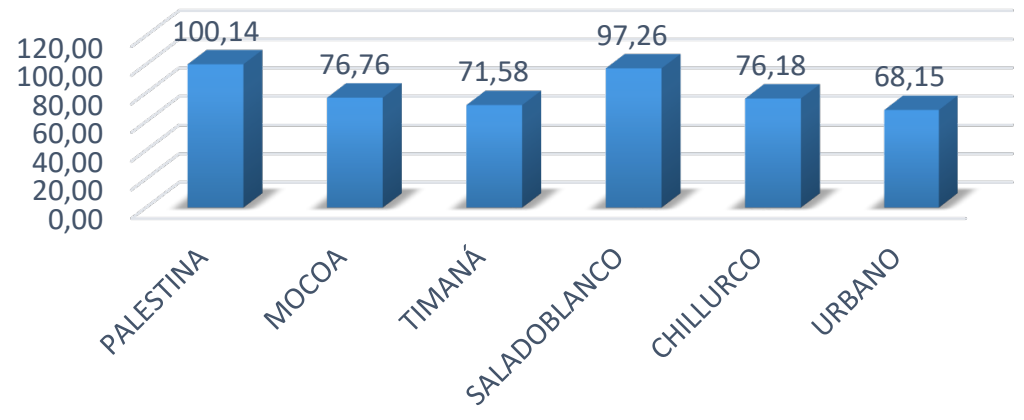

Figura 5. Biomasa por ruta Ton/ha.

Fuente: Autores (2018) 
En la figura se muestran los resultados de biomasa seca en Ton/ha para cada ruta estudiada, resaltando el gran aporte de la ruta Palestina, la cual supera las 100 Ton/ha.

\section{DISCUSIÓN Y ANÁLISIS}

El aporte en promedio de biomasa total más sobresaliente se dio en la ruta Palestina, aporta en promedio 100,14 Ton/ha. En comparación con las demás rutas, cabe señalar que en esta ruta se presenta una mayor cantidad de guaduales en relación con las otras rutas evaluadas, y además, con buenas condiciones ambientales y buenos estados de manejo y cuidado de los guaduales.

El resultado estimado sobre el valor total de biomasa aérea seca brindada por la Guadua angustifolia Kunth, para el municipio de Pitalito Huila, sobre la cuenca del río Guarapas, es de 28692,20 toneladas, este dato resulta del promedio general de biomasa seca por hectárea, y de la extensión total de guadua que hay para el municipio de Pitalito Huila, que es de 347,9 hectáreas. Sin embargo, no se cuenta con la extensión de cada área del municipio, para obtener un dato más preciso, ya que una de las áreas podría tener un porcentaje de extensión de guadua mayor que otro, por ejemplo, la ruta Palestina, lo que podría variar el resultado final del presente trabajo.

En el caso de las comparaciones realizadas con el estudio de Ríos (S.f.), permite deducir que la guadua existente en la cuenca hidrográfica del río Guarapas en el municipio de Pitalito Huila presenta una notoria desventaja frente al contenido de humedad, de más del $20 \%$ frente a los resultados obtenidos por Ríos, lo cual puede deberse a la ubicación geográfica, aunque esa es una cuestión difícil de resolver o de dar hipótesis por medio de los resultados obtenidos, ya que sería necesario realizar estudios geográficos, de suelos, flora y fauna asociada, entre otros que pueden afectar esta variable. Por otra parte, la comparación realizada respecto al volumen en Ton/ha de biomasa aportada por cada componente evaluado, permitió observar que el promedio obtenido en el presente estudio es muy bajo respecto al obtenido en el estudio realizado por Ríos, aunque esto puede deberse a que en el presente estudio no se tuvo en cuenta la variable de edad, y como demuestra Ríos, la Guadua angustifolia presenta una mayor producción de biomasa a la edad de 4, 5 y 6 años, lo cual sería una variable importante a tomar en cuenta en futuras investigaciones.

Del resultado de aporte de biomasa seca por hectárea, que es de 82,47Ton/ha en promedio, se puede estimar la cantidad de biomasa seca total que aporta la guadua del municipio de Pitalito, incluyendo rizomas y raíces, tomando en 
cuenta que, según (Camargo, 2010), la biomasa aérea contiene aproximadamente un $74,6 \%$ de la biomasa total de la guadua, por lo tanto, se puede estimar que la Guadua angustifolia Kunth ubicada sobre la cuenca hidrográfica del río Guarapas en el municipio de Pitalito, aporta en promedio 110,54 Ton/ha.

\section{CONCLUSIONES}

Se analizó y se estimó la biomasa aérea recolectada de los individuos seleccionados, obteniendo un valor promedio de 82 Ton/ha para todos los rodales estudiados, notándose una gran variabilidad entre los valores obtenidos en las diferentes rutas, respecto al contenido de humedad y volumen de biomasa, lo cual permite concluir que la Guadua angustifolia Kunth presente sobre la cuenca hidrográfica del río Guarapas para el municipio de Pitalito, presenta diferentes condiciones en diferentes sectores, aunque pertenezcan a una misma cuenca, lo que permitiría realizar estudios similares a éste pero evaluando zonas más pequeñas, o incluyendo nuevas variables, como las edades de las guaduas, áreas de los rodales, tiempos de corte, proximidad a centros poblados o vías transitadas, y demás factores que puedan influenciar en los resultados.

El presente proyecto es base para posteriores investigaciones en la región, las cuales se espera que aporten y/o contribuyan a la conservación, sostenibilidad y adecuado aprovechamiento de los recursos naturales.

Los resultados finales del presente trabajo, permiten identificar el gran aporte a nivel ambiental que la Guadua angustifolia Kunth brinda al municipio de Pitalito, principalmente a las fuentes hídricas, ya que generalmente se presenta cerca a fuentes hídricas, y además, permite identificar algunos fallos frente al tratamiento de esta especie, la cual necesita de cuidado y de aprovechamiento periódico para poder extenderse y desarrollarse adecuadamente, procesos que actualmente la legislación nacional y regional tienen muy condicionados, lo que evita que los propietarios de predios que poseen guadua no realicen adecuadas técnicas silviculturales, que causa que la guadua no pueda desarrollarse en un ambiente totalmente adecuado, y esto conlleva a que genere menor cantidad de biomasa, por lo tanto reduzca su capacidad de captura de carbono, entre otras consecuencias.

Es notable el potencial que brinda la guadua en el aspecto ambiental (Gamboa, 2015), económico y social, ya que, además de sus servicios ecosistémicos (corredor, Fonseca \& Páez, 2012), genera un promedio de 82 Ton/ha como se 
demostró en el presente trabajo, además su aprovechamiento se puede dar a una edad aproximada de 6 años, un tiempo relativamente muy corto frente a las especies maderables, también sirve como sustituto de la madera, propiedad que puede ser utilizada para la protección de especies maderables en vía de extinción, su utilización se puede hacer de forma manual o por medio de equipos industriales, es decir, es muy manejable y se le puede dar diversos usos, puede generar empleo para la región y promover la economía y el mercado regional y nacional, y promover los productos nacionales ante los importados.

En este marco de investigaciones se considera que la biotecnología aplicada al sector agroindustrial con la especie guadua (Guadua spp.) es muy importante ya que representa hoy en día un mayor desarrollo para el país en comparación a otros subsectores; en conclusión, muestra que hay una alta diversidad genética y debido a esta variabilidad se puede pensar en un mejoramiento genético de esta especie guadua; se puede visualizar de forma positiva para un futuro, además de ser una aplicación sustentable de la biotecnología agropecuaria enmarcada dentro de las tendencias mundiales, no obstante se requiere de esfuerzos significativos en investigación básica y aplicada que coloquen a Colombia a la vanguardia del desarrollo biotecnológico agropecuario. (Montenegro Gómez, S., \& Hernández Ossa, Y. 2015).

\section{RECOMENDACIONES}

- En base a los resultados se evidencia la necesidad de avanzar en la realización de posteriores trabajos de investigación con relación a la Guadua angustifolia Kunth, con el fin de determinar la cuantificación de carbono de la misma.

- De igual forma, se recomienda la realización de estudios similares, evaluando el aporte de biomasa en diferentes edades, y en diferentes subtipos de esta especie vegetal.

- $\quad$ Otro punto importante a realizarse en un futuro cercano, es el desarrollo de estudios similares, con especies vegetales abundantes en la región, como el lulo, el maíz, etc., buscando la cuantificación total de biomasa seca y de captura de carbono aportada en el municipio de Pitalito Huila. 


\section{BIBLIOGRAFÍA}

Agroempresarial S.A. (2012). Plan de Aprovechamiento y manejo de flora silvestre: Guadua, en el concepto del núcleo forestal productivo. Pitalito, Huila, Colombia: Agroempresarial S.A. Recuperado el 29 de Septiembre de 2017.

Arango, A. M. (2011). Posibilidades de la guadua para la mitigación del cambio climático caso: Eje Cafetero Colombiano. Pereira, Colombia: Universidad Tecnológica de Pereira. Recuperado el 28 de Septiembre de 2017.

Bambú. (2 de diciembre de 2015). ¿Cuántas especies de bambú existen? Recuperado el 12 de marzo de 2018, de Bambú: http://bambuver.blogspot.com/2015/12/cuantas-especies-de-bambuexisten.html

Bárbaro, G. (2007). Transformación e industrialización del Bambú. Barcelona, España: Arquitectura del paisaje. Obtenido de http://www.horticom.com/revistasonline/gej/bp155/08 15.pdf.

Bonilla Morales, M., \& Caetano, C. (2013). Inventario y valoración de la flora utilizada por la vereda Santa Teresa, Palmira (Valle del Cauca). Revista de Investigación Agraria y Ambiental, 4(1), 89-99. doi:https://doi.org/10.22490/21456453.985

Camargo, J. C., Rodríguez, J. A., Arango, A. M. (2010). Crecimiento y fijación de carbono en una plantación de guadua en la zona cafetera de Colombia. Recuperado el 23 de 03 de 2018, de Grupo de investigación GATA:

http://repositorio.bibliotecaorton.catie.ac.cr/bitstream/handle/11554/5 987/13. Camargo.pdf

Camargo, J. C., Rodríguez, J. A., \&amp; Arango, Á. M. (2010). Bosques de guadua del Eje Cafetero de Colombia: oportunidades para su inclusión en el mercado voluntario de carbono y en el Programa REDD+. Colombia: Comunicación Técnica. Recuperado el 22 de Noviembre de 2017, 
http://bco.catie.ac.cr/portalrevistas/index.php/RRNA/article/view/97/1 $\underline{76}$

Cambio climático.org. (S.f.). Efectos del cambio climático. Recuperado el 8 de Febrero de 2018, de Cambio climático.org: http://www.cambioclimatico.org/tema/efectos-del-cambio-climatico

Chará, J., Giraldo, L. P., Chará, M. y Ximena, G. (2010). Beneficios de los corredores ribereños de Guadua angustifolia en la protección de corredores acuáticos en la Ecorregión a 80 Cafetera de Colombia. 2. Efectos sobre la escorrentía y captura de nutrientes. Recursos Naturales y Ambiente, (61), 60-66.

Corredor Camargo, E., Fonseca Carreño, J., \& Páez Barón, E. (2012). Los servicios ecosistémicos de regulación: tendencias e impacto en el bienestar humano. Revista de Investigación Agraria y Ambiental, 3(1), 77-83. doi:https://doi.org/10.22490/21456453.936 (corredor, Fonseca y Páez, 2012)

Cuellar B. Adelaida, Montealegre T. William, Méndez P. Nelly M. (2016). Formulación Plan prospectivo y estratégico para la consolidación de la cadena productiva de la Guadua en la zona sur del Huila Colombia.

Duarte, L. A., Arana-Gutiérrez, A. D. (2016). Medio ambiente y naturaleza al servicio del capital corporativo transnacional. Revista Criterio Libre Jurídico. 13(1), 85-103 Recuperado de: http://revistasojs.unilibrecali.edu.co/index.php/rclj/article/download/5 $46 / 865$

Ecohabitar. (2015). La guadua: una maravilla natural de grandes bondades y prometedor futuro. Obtenido de http://www.ecohabitar.org/la-guaduauna-maravilla-natural-de-grandes-bondades-y-prometedor-futuro/

Gamboa García, D. (2015). Valoración de impactos ecológicos por minería de oro en río Guabas, Valle del Cauca, Colombia. Revista de Investigación $\begin{array}{lllll}\text { Agraria y Ambiental, 6(2), } 243 \quad \text { - } & 254 .\end{array}$ doi:https://doi.org/10.22490/21456453.1420

Giraldo Díaz, R., \& Valencia T., F. (2010). Evaluación de la sustentabilidad ambiental de tres sistemas de producción agropecuarios, en el corregimiento Bolo San Isidro, Palmira (Valle del Cauca). Revista de 
Investigación Agraria y Ambiental, 1(2), 7-17. doi:https://doi.org/10.22490/21456453.900

González Zárate Mequeas. (2008). Tesis de grado Maestría "Estimación de la biomasa aérea y la captura de carbono en regeneración natural de Pinus maximinoi H. E. Moore, Pinus oocarpa var. Ochoterenai Mtz. y Quercus sp. En el norte del Estado de Chiapas, México.

González, W. F. \& Vargas, M. R. (2015). Acumulación y predicción de biomasa y carbono en plantaciones de bambú en Costa Rica. Bogotá, Colombia: Ambiente y Desarrollo. Recuperado el 28 de septiembre de 2017, obtenido de http://dx.doi.org/10.11144/Javeriana.ayd20-38.apbc doi: 10.11144/Javeriana.up14-4.ayd20-38.apbc

Herrera, E. G. (2008). Bienes y servicios ambientales de la guadua en Colombia (Guadua angustifolia Kunth). Sigguadua. Recuperado el 22 de Noviembre de 2017, de http://www.sigguadua.gov.co/sites/default/files/archivos/bienes y ser vicios guadua.pdf

INYUMACIZO, G. D. (2012). Promoción, innovación y desarrollo industrial de la guadua (Guadua angustifolia Kunth), en la cuenca hidrográfica del río Guarapas, departamento del Huila Colombia. Pitalito.

IPCC. (2007). Informe de síntesis cambio climático 2007. Grupo Intergubernamental de Expertos sobre el Cambio Climático. Recuperado el 23 de Noviembre de 2017, de https://www.ipcc.ch/pdf/assessmentreport/ar4/syr/ar4_syr_sp.pdf

Matos de Oliveira, A. (2014). Crítica ecológica al concepto de Desarrollo y nuevas alternativas desde América Latina. Revista de Investigación Agraria y Ambiental, 5(2), $41 \quad$ - 53. doi:https://doi.org/10.22490/21456453.1325

Méndez Pedroza, Nelly María (2014). Trabajo de grado Doctorado "Diagnóstico de guaduales y propuesta de un modelo de ordenamiento forestal sostenible productivo para el manejo e industrialización de la guadua (Guadua angustifolia Benth), con participación comunitaria en la Cuenca hidrográfica del río Guarapas, zona sur del departamento del Huila, Colombia. 
Mendoza Velásquez, S., Cano Muñoz, J. \& Rojas Sánchez, F. (2015). Acción comunitaria frente al fenómeno del cambio climático, en el páramo de la región del Guavio, Cundinamarca, Colombia. Revista de Investigación Agraria y Ambiental, 6(1), $265 \quad$ - 279. doi:https://doi.org/10.22490/21456453.1286

Mónaco, N., Rosa, M. J., Santa, V., Autrán, V., \& Heguiabehere, A. (2015). Utilización de estimadores para determinación de biomasa a campo. Argentina: European Scientific Journal. Recuperado el 23 de Noviembre de 2018, de https://eujournal.org/index.php/esj/article/download/6653/6390

Montealegre Torres, William Ignacio (2014). Trabajo de grado maestría "Formulación del plan prospectivo y estratégico para la consolidación de la cadena productiva de la guadua en la cuenca hidrográfica del río Guarapas, departamento del Huila, Colombia". Recuperado el 22 de noviembre de 2017. UNAD.

Montenegro Gómez, S., \& Hernández Ossa, Y. (2015). Biotecnología aplicada al desarrollo agropecuario colombiano. Revista de Investigación Agraria $\begin{array}{lllll}\text { y Ambiental, } & 6(2), & 97 & \end{array}$ doi:https://doi.org/10.22490/21456453.1408

Montes, J. y Sánchez, W. (2016). Relación Sociedad Civil, Estado y Economía en el mundo contemporáneo. Revista Criterio Libre Jurídico. 13(2), 3649 http://dx.doi.org/10.18041/crilibjur.2016.v13n2.26203

Riaño, N. M., Londoño, X., López, Y., \& Gómez, J. H. (2002). Plant growth and biomass distribution on Guadua angustifolia Kunth in relation to ageing in the Valle del Cauca - Colombia. Colombia: Bamboo Science and Culture. Recuperado el 10 de Julio de 2018, de http://www.maderinsa.com/guadua/fijacion.pdf

Ríos, H. C. (S.f.). Biomasa y Atrapamiento de carbono en Bambú Guadua. Colombia - México: Bambuguaduapremier.com. Recuperado el 28 de Septiembre de 2017 de https://es.scribd.com/document/325104417/Biomasa-y-Atrapamientode-carbono-en-Bambu-Guadua-pdf 
Teneche, G. (s.f.). GUADUA Y BAMBU COLOMBIA (GBC) Guadua angustifolia Kunth. Obtenido de https://guaduabambucolombia.com/

Vinasco Guzmán, M. (2017). Marco teórico para la construcción de una propuesta de turismo rural comunitario. Revista de Investigación $\begin{array}{llllll}\text { Agraria } & y & \text { Ambiental, } & 8(1), & 95 & -\end{array}$ doi:https://doi.org/10.22490/21456453.1841

Zapata Galvis, J., \& Llano Franco, J. (2014). Perspectiva interdisciplinaria del ordenamiento alimentario y derecho del consumidor en Argentina. Revista de Investigación Agraria y Ambiental, 5(2), 99 - 114. doi:https://doi.org/10.22490/21456453.1329 